\title{
The structure of large non-trivial $t$-intersecting families for finite sets
}

\author{
Mengyu $\mathrm{Cao}^{* 1,2}$, Benjian $\mathrm{Lv}^{\dagger 1}$, and Kaishun Wang ${ }^{\ddagger 1}$ \\ ${ }^{1}$ Laboratory of Mathematics and Complex Systems (Ministry of Education), School of \\ Mathematical Sciences, Beijing Normal University, Beijing 100875, China \\ ${ }^{2}$ Department of Mathematical Sciences, Tsinghua University, Beijing 100084, China
}

\begin{abstract}
In this paper, we describe the structure of maximal non-trivial uniform $t$-intersecting families with large size for finite sets. In the special case when $t=1$, our result gives rise to Kostochka and Mubayi's result in 2017.
\end{abstract}

AMS classification: 05D05, 05A10

Key words: Erdős-Ko-Rado Theorem; Hilton-Milner Theorem; $t$-intersecting family; $t$ covering number

\section{Introduction}

Let $n$ and $k$ be integers with $1 \leq k \leq n$. Write $[n]=\{1,2, \ldots, n\}$ and denote by $\left(\begin{array}{c}{[n]} \\ k\end{array}\right)$ the family of all $k$-subsets of $[n]$. For any positive integer $t$, a family $\mathcal{F} \subseteq\left(\begin{array}{c}{[n]} \\ k\end{array}\right)$ is said to be $t$-intersecting if $|A \cap B| \geq t$ for all $A, B \in \mathcal{F}$. A $t$-intersecting family is called trivial if all its members contain a common specified $t$-subset of $[n]$, and non-trivial otherwise.

The famous Erdős-Ko-Rado Theorem gives the maximum size of a $t$-intersecting family and shows further that any $t$-intersecting family with maximum size is a trivial family consisting of all $k$-subsets that contain a fixed $t$-subset of $[n]$ for $n>n_{0}(k, t)$ [4]. It is known that the smallest possible such function $n_{0}(k, t)$ is $(t+1)(k-t+1)$. This was proved by Frankl [5] for $t \geq 15$ and subsequently determined by Wilson [18] for all $t$. In [5], Frankl also made a conjecture on the maximum size of a $t$-intersecting family of $k$-subsets of $[n]$ for any positive integers $t, k$ and $n$. This conjecture was partially proved by Frankl and Füredi in [10] and completely settled by Ahlswede and Khachatrian in [2].

Determining the structure of non-trivial $t$-intersecting families of $k$-subsets of $[n]$ with maximum size was a long-standing problem. The first result is the Hilton-Milner Theorem [16] which describes the structure of such families for $t=1$. A significant step was taken in [6]

\footnotetext{
${ }^{*}$ E-mail: caomengyu@mail.bnu.edu.cn

${ }^{\dagger}$ Corresponding author. E-mail: bjlv@bnu.edu.cn

${ }^{\ddagger}$ E-mail: wangks@bnu.edu.cn
} 
by Frankl, who determined such families for $t \geq 2$ and $n>n_{1}(k, t)$. In [9], Frankl and Füredi gave a short and elegant proof for the Hilton-Milner Theorem by using the shifting technique, and also asked whether $n_{1}(k, t)<c k t$ holds. Ahlswede and Khachatrian [1] answered this question and gave a complete result on non-trivial intersection problems for finite sets.

Recently, other maximal non-trivial 1-intersecting families with large size have been studied. In [15], Han and Kohayakawa determined the structure of the third largest maximal 1 -intersecting families of $k$-subsets of $[n]$ with $6 \leq 2 k<n$. They also mentioned that it would be natural to investigate such problem for $t$-intersecting families. In [17], Kostochka and Mubayi described the structure of 1-intersecting families of $k$-subsets of $[n]$ for large $n$ whose size is quite a bit smaller than the bound $\left(\begin{array}{c}n-1 \\ k-1\end{array}\right)$ given by the Erdős-Ko-Rado Theorem.

Define the $t$-covering number $\tau_{t}(\mathcal{F})$ of a family $\mathcal{F} \subseteq\left(\begin{array}{c}{[n]} \\ k\end{array}\right)$ to be the minimum size of a subset $T$ of $[n]$ such that $|T \cap F| \geq t$ for any $F \in \mathcal{F}$. Let $\mathcal{F} \subseteq\left(\begin{array}{c}{[n]} \\ k\end{array}\right)$ be any $t$-intersecting family. Note that $t \leq \tau_{t}(\mathcal{F}) \leq k$, and $\mathcal{F}$ is trivial if $\tau_{t}(\mathcal{F})=t$. In [6], in order to describe the structure of non-trivial $t$-intersecting families with maximum size for $t \geq 2$, Frankl defined the family $\mathcal{F}^{(t)}$ and the base $\mathcal{B}$ of $\mathcal{F}$. It is straightforward to verify that the $t$-covering number of a maximal non-trivial $t$-intersecting family equals to $\ell_{1}$ in the decomposition of its base $\mathcal{B}$. Observe that when $t=1$ that is the well-known covering number of an intersecting family. We refer the readers to $[7,11,12,13]$ for more results about the covering number.

In this paper, we consider maximal non-trivial $t$-intersecting families with large size for any positive integer $t$. If $t=k-1$, it is well known that any maximal non-trivial $(k-1)$ intersecting family is a collection of all $k$-subsets containing a fixed $(k-1)$-subset or a collection of all $k$-subsets contained in a fixed $(k+1)$-subset. Thus, we only consider the case with $1 \leq t \leq k-2$. To present our result let us first introduce the following two constructions of $t$-intersecting families of $k$-subsets of $[n]$.

Family I. Let $X, M$ and $C$ be three subsets of $[n]$ such that $X \subseteq M \subseteq C,|X|=t,|M|=k$ and $|C|=c$, where $c \in\{k+1, k+2, \ldots, 2 k-t, n\}$. Denote

$$
\mathcal{H}_{1}(X, M, C)=\mathcal{A}(X, M) \cup \mathcal{B}(X, M, C) \cup \mathcal{C}(X, M, C),
$$

where

$$
\begin{aligned}
\mathcal{A}(X, M) & =\left\{F \in\left(\begin{array}{c}
{[n]} \\
k
\end{array}\right)|X \subseteq F,| F \cap M \mid \geq t+1\right\}, \\
\mathcal{B}(X, M, C) & =\left\{F \in\left(\begin{array}{c}
{[n]} \\
k
\end{array}\right)|F \cap M=X,| F \cap C \mid=c-k+t\right\}, \\
\mathcal{C}(X, M, C) & =\left\{F \in\left(\begin{array}{c}
C \\
k
\end{array}\right)|| F \cap X|=t-1,| F \cap M \mid=k-1\right\} .
\end{aligned}
$$

Family II. Let $Z$ be a $(t+2)$-subset of $[n]$. Define

$$
\mathcal{H}_{2}(Z)=\left\{F \in\left(\begin{array}{c}
{[n]} \\
k
\end{array}\right)|| F \cap Z \mid \geq t+1\right\} .
$$

It is straightforward to verify that Families I and II are $t$-intersecting families with $t$ covering number $t+1$. Observe that the size of each this family only depends on $|X|,|M|$, $|C|$ and $|Z|$. Let $h_{1}(t, k, c)=\left|\mathcal{H}_{1}(X, M, C)\right|$, where $c=|C| \in\{k+1, k+2, \ldots, 2 k-t, n\}$; $h_{2}(t+2)=\left|\mathcal{H}_{2}(Z)\right|$. 
Remark 1 Suppose $X, M$ and $C$ are three subsets of $[n]$ satisfying the condition in Family I. If $|C|=k+1$, then

$$
\mathcal{H}_{1}(X, M, C)=\left\{F \in\left(\begin{array}{c}
{[n]} \\
k
\end{array}\right)|X \subseteq F,| F \cap C \mid \geq t+1\right\} \cup\left(\begin{array}{l}
C \\
k
\end{array}\right) .
$$

If $t=k-2$, then $\mathcal{H}_{1}(X, M,[n])=\mathcal{H}_{2}(M)$ and $h_{1}(k-2, k, n)=h_{2}(k)$.

Our main result describes the structure of all maximal non-trivial uniform $t$-intersecting families with large size for finite sets.

Theorem 1.1 Let $1 \leq t \leq k-2$, and $\max \left\{\left(\begin{array}{c}t+2 \\ 2\end{array}\right), \frac{k-t+2}{2}\right\} \cdot(k-t+1)^{2}+t \leq n$. If $\mathcal{F} \subseteq\left(\begin{array}{c}{[n]} \\ k\end{array}\right)$ is a maximal non-trivial t-intersecting family with

$$
|\mathcal{F}| \geq(k-t)\left(\begin{array}{l}
n-t-1 \\
k-t-1
\end{array}\right)-\left(\begin{array}{c}
k-t \\
2
\end{array}\right)\left(\begin{array}{l}
n-t-2 \\
k-t-2
\end{array}\right)
$$

then one of the following holds.

(i) $\mathcal{F}=\mathcal{H}_{1}(X, M, C)$ for some t-subset $X, k$-subset $M$ and $c$-subset $C$ of $[n]$, where $c \in\{k+1, k+2, \ldots, 2 k-t, n\}$.

(ii) $\mathcal{F}=\mathcal{H}_{2}(Z)$ for some $(t+2)$-subset $Z$ of $[n]$, and $\frac{k}{2}-1 \leq t \leq k-2$.

In the special case when $t=1$, Theorem 1.1 gives rise to Theorem 7 in [17]. From Lemmas 2.6, 2.7, 2.8 and 2.9, one can determine the sequence of these families according to their sizes.

The rest of this paper is organized as follows. In the next section we will give some properties of the maximal $t$-intersecting families with $t$-covering number $t+1$, and prove a number of inequalities for the sizes of Families I and II. In Section 3 we will prove some upper bounds for the sizes of non-trivial $t$-intersecting families using their $t$-covering number. After these preparations we will prove Theorem 1.1 in Section 4.

\section{$2 t$-intersecting families with $t$-covering number $t+1$}

In this section, we will give some properties of the maximal $t$-intersecting families with $t$ covering number $t+1$ and prove a number of inequalities for the sizes of Families I and II.

Assumption 1 Let $1 \leq t \leq k-2$ and $2 k \leq n$, let $\mathcal{F} \subseteq\left(\begin{array}{c}{[n]} \\ k\end{array}\right)$ be a maximal $t$-intersecting family with $\tau_{t}(\mathcal{F})=t+1$. Define

$$
\mathcal{T}=\left\{T \in\left(\begin{array}{c}
{[n]} \\
t+1
\end{array}\right)|| T \cap F \mid \geq t \text { for any } F \in \mathcal{F}\right\} .
$$

Observe that the $\mathcal{T}$ in Assumption 1 is the family $\mathcal{B}_{\ell_{1}}$ with $\ell_{1}=t+1$ in the decomposition of the base $\mathcal{B}$ of $\mathcal{F}$ in [6]. Applying the method in the proof of Theorem 1 in [6], we firstly give several properties of the maximal $t$-intersecting families with $t$-covering number $t+1$. 
Lemma 2.1 Let $n, k, t, \mathcal{F}$ and $\mathcal{T}$ be as in Assumption 1. Then $\mathcal{T}$ is a t-intersecting family with $t \leq \tau_{t}(\mathcal{T}) \leq t+1$. Moreover, the following hold.

(i) If $\tau_{t}(\mathcal{T})=t$, then there exist a $t$-subset $X$ and an l-subset $M$ of $[n]$ with $X \subseteq M$ and $t+1 \leq l \leq k+1$ such that

$$
\mathcal{T}=\left\{T \in\left(\begin{array}{c}
M \\
t+1
\end{array}\right) \mid X \subseteq T\right\}
$$

(ii) If $\tau_{t}(\mathcal{T})=t+1$, then there exists a $(t+2)$-subset $Z$ of $[n]$ such that $\mathcal{T}=\left(\begin{array}{c}Z \\ t+1\end{array}\right)$.

Proof. For any $T \in \mathcal{T}$, by maximality of $\mathcal{F}, \mathcal{F}$ contains all $k$-subsets of $[n]$ containing $T$. For any $T_{1}, T_{2} \in \mathcal{T}$, if $\left|T_{1} \cap T_{2}\right|<t$, then there must exist $F_{1}, F_{2} \in \mathcal{F}$ such that $T_{1} \subseteq F_{1}$, $T_{2} \subseteq F_{2}$ and $\left|F_{1} \cap F_{2}\right|<t$ from $2 k \leq n$. That is impossible as $\mathcal{F}$ is maximal $t$-intersecting. Hence $\left|T_{1} \cap T_{2}\right| \geq t$, and $\mathcal{T} \subseteq\left(\begin{array}{c}{[n]} \\ t+1\end{array}\right)$ is a $t$-intersecting family with $t \leq \tau_{t}(\mathcal{T}) \leq t+1$.

(i) Suppose that $\tau_{t}(\mathcal{T})=t$. Then there exists a $t$-subset $X$ of $[n]$ such that $X$ is contained in every $(t+1)$-subset in $\mathcal{T}$. Assume that $M=\cup_{T \in \mathcal{T}} T$ and $|M|=l$. It suffices to prove (1) and $t+1 \leq|M| \leq k+1$. Since $\tau_{t}(\mathcal{F})=t+1$, we have $\mathcal{F} \backslash \mathcal{F}_{X} \neq \emptyset$. Let $F^{\prime}$ be any $k$-subset in $\mathcal{F} \backslash \mathcal{F}_{X}$. Observe that $\left|X \cap F^{\prime}\right| \leq t-1$. For any $T \in \mathcal{T}$, since $X \subseteq T$ and $\left|T \cap F^{\prime}\right| \geq t$, we have $\left|X \cap F^{\prime}\right|=t-1$ and $\left|T \cap\left(X \cup F^{\prime}\right)\right| \geq t+1$, which imply that $\left|X \cup F^{\prime}\right|=k+1$ and $T \subseteq X \cup F^{\prime}$. Hence $M=\cup_{T \in \mathcal{T}} T \subseteq X \cup F^{\prime}$ and $t+1 \leq l \leq k+1$. It is clear that $\mathcal{T} \subseteq\left\{T \in\left(\begin{array}{c}M \\ t+1\end{array}\right) \mid X \subseteq T\right\}$. Let $T^{\prime}$ be any $(t+1)$-subset of $M$ with $X \subseteq T^{\prime}$. For any $F \in \mathcal{F}$, if $X \subseteq F$, then $\left|T^{\prime} \cap F\right| \geq t$; if $X \nsubseteq F$, by above discussion, then $T^{\prime} \subseteq X \cup F$, which implies that $\left|T^{\prime} \cap F\right| \geq t$ from $|X \cup F|=k+1$. Hence $T^{\prime} \in \mathcal{T}$ and (1) is proved.

(ii) Suppose that $\tau_{t}(\mathcal{T})=t+1$. Let $A, B, C \in \mathcal{T}$ be distinct subsets such that $A \cap B$, $A \cap C$ and $B \cap C$ are pair-wise distinct. Since $\mathcal{T}$ is $t$-intersecting, we have $|A \cap B|=|A \cap C|=$ $|B \cap C|=t$, which implies that $C=(A \cap C) \cup(B \cap C) \subseteq A \cup B$ from $|C|=t+1$. Hence, we get $A \cup C \subseteq A \cup B$ and $B \cup C \subseteq A \cup B$, which imply that $A \cup B=A \cup C=B \cup C$.

Since $\tau_{t}(\mathcal{T})=t+1$, there exist three distinct subsets $T_{1}, T_{2}, T_{3} \in \mathcal{T}$ such that $T_{1} \cap T_{2}$, $T_{1} \cap T_{3}$ and $T_{2} \cap T_{3}$ are pair-wise distinct. For any $T \in \mathcal{T} \backslash\left\{T_{1}, T_{2}, T_{3}\right\}$, if $T \cap T_{1}=$ $T \cap T_{2}=T \cap T_{3}$, then $\left|T \cap T_{1}\right|=t, T \cap T_{1} \subseteq T_{2}$ and $T \cap T_{1} \subseteq T_{3}$, which imply that $T \cap T_{1}=T_{1} \cap T_{2}=T_{1} \cap T_{3}$, a contradiction. Hence, there exist $T_{i}, T_{j} \in\left\{T_{1}, T_{2}, T_{3}\right\}$ such that $T \cap T_{i} \neq T \cap T_{j}$, and $T=\left(T \cap T_{i}\right) \cup\left(T \cap T_{j}\right) \subseteq T_{1} \cup T_{2}=T_{1} \cup T_{3}=T_{2} \cup T_{3}$.

Let $Z=T_{1} \cup T_{2}$. Then $\mathcal{T} \subseteq\left(\begin{array}{c}Z \\ t+1\end{array}\right)$. In the following, we show that $\left(\begin{array}{c}Z \\ t+1\end{array}\right) \subseteq \mathcal{T}$. For any $F \in \mathcal{F}$, if $F \cap T_{1}=F \cap T_{2}=F \cap T_{3}$, then $F \cap T_{1} \subseteq T_{i}$ for any $i \in\{1,2,3\}$. That is impossible as $T_{1} \cap T_{2}, T_{1} \cap T_{3}$ and $T_{2} \cap T_{3}$ are pair-wise distinct and $\left|F \cap T_{1}\right| \geq t$. Hence there exist $T_{i}, T_{j} \in\left\{T_{1}, T_{2}, T_{3}\right\}$ such that $F \cap T_{i} \neq F \cap T_{j}$, implying that $|F \cap Z| \geq t+1$. So for any $F \in \mathcal{F}$ and $T^{\prime} \in\left(\begin{array}{c}Z \\ t+1\end{array}\right)$, we have $\left|F \cap T^{\prime}\right| \geq t$. Therefore, we have $\mathcal{T}=\left(\begin{array}{c}Z \\ t+1\end{array}\right)$ as desired.

Lemma 2.2 Let $n, k, t, \mathcal{F}$ and $\mathcal{T}$ be as in Assumption 1, and set $M=\cup_{T \in \mathcal{T}} T$. Suppose that $\tau_{t}(\mathcal{T})=t,|M|=k+1$ and $X$ is a $t$-subset of $[n]$ which is contained in each $T \in \mathcal{T}$. Then $\mathcal{F}=\left\{F \in\left(\begin{array}{c}{[n]} \\ k\end{array}\right)|X \subseteq F|, F \cap M \mid \geq t+1\right\} \cup\left(\begin{array}{c}M \\ k\end{array}\right)$.

Proof. It follows from the proof of Lemma 2.1 that, for any $F \in \mathcal{F} \backslash \mathcal{F}_{X}$, we have $M=F \cup X$, which implies that $F \in\left(\begin{array}{c}M \\ k\end{array}\right)$. Let $\mathcal{A}^{\prime}=\left\{F \in\left(\begin{array}{c}{[n]} \\ k\end{array}\right)|X \subseteq F|, F \cap M \mid \geq t+1\right\}$ and $F^{\prime}$ be a fixed $k$-subset in $\mathcal{F} \backslash \mathcal{F}_{X}$. For any $F \in \mathcal{F}_{X}$, since $\left|F \cap F^{\prime}\right| \geq t,\left|F^{\prime} \cap X\right| \leq t-1$ and $M=F^{\prime} \cup X$, 
we have $|F \cap M| \geq t+1$, which implies that $\mathcal{F}_{X} \subseteq \mathcal{A}^{\prime}$. Note that $\mathcal{A}^{\prime} \cup\left(\begin{array}{c}M \\ k\end{array}\right)$ is a $t$-intersecting family. By the maximality of $\mathcal{F}$, we have $\mathcal{F}=\mathcal{A}^{\prime} \cup\left(\begin{array}{c}M \\ k\end{array}\right)$.

By Remark 1 , if $\mathcal{F}$ is a maximal $t$-intersecting family satisfying the conditions in Lemma 2.2, then $\mathcal{F}=\mathcal{H}_{1}(X, Y, M)$ for any $Y \in\left(\begin{array}{c}M \\ k\end{array}\right)$ with $X \subseteq Y$.

Lemma 2.3 Let $n, k, t, \mathcal{F}$ and $\mathcal{T}$ be as in Assumption 1. Suppose that $\tau_{t}(\mathcal{T})=t$, and $X$ is a t-subset of $[n]$ which is contained in each $T \in \mathcal{T}$. Set $M=\cup_{T \in \mathcal{T}} T$ and $C=M \cup$ $\left(\cup_{F \in \mathcal{F} \backslash \mathcal{F}_{X}} F\right)$. Assume that $|M|=k$ and $|C|=c$. Then either $k+2 \leq c \leq 2 k-t$ or $c=n$. Moreover, the following hold.

(i) If $k+2 \leq c \leq 2 k-t$, then $\mathcal{F}=\mathcal{H}_{1}(X, M, C)$.

(ii) If $c=n$, then $t \neq k-2$ and $\mathcal{F}=\mathcal{H}_{1}(X, M,[n])$.

Proof. By the proof of Lemma 2.1, for any $F \in \mathcal{F} \backslash \mathcal{F}_{X}$, we have $|F \cap X|=t-1$ and $M \subseteq X \cup F$, which imply $|F \cap M|=k-1$ from $X \subseteq M$. Choose $F_{1} \in \mathcal{F} \backslash \mathcal{F}_{X}$. Then $\left|F_{1} \cup M\right|=k+1$. If $c>k+1$, then there exists $F_{2} \in \mathcal{F} \backslash \mathcal{F}_{X}$ such that $F_{2} \nsubseteq F_{1} \cup M$, which implies that $F_{2} \cap\left(F_{1} \cup M\right)=F_{2} \cap M$. Similarly, if $c>k+2$, then there exists $F_{3} \in \mathcal{F} \backslash \mathcal{F}_{X}$ such that $F_{3} \nsubseteq F_{1} \cup F_{2} \cup M$, which implies that $F_{3} \cap\left(F_{1} \cup F_{2} \cup M\right)=F_{3} \cap M$. By mathematical induction, we can get $F_{1}, F_{2}, \ldots, F_{c-k} \in \mathcal{F} \backslash \mathcal{F}_{X}$ such that $F_{i} \cap\left(M \cup\left(\cup_{j=1}^{i-1} F_{j}\right)\right)=F_{i} \cap M$ for any $i \in\{1,2, \ldots, c-k\}$. If there exists $F^{\prime} \in \mathcal{F}$ such that $F^{\prime} \cap M=X$, then for any $i \in\{1,2, \ldots, c-k\}$, there exists $y_{i} \in F_{i} \backslash M$ such that $y_{i} \in F^{\prime}$ from $\left|F^{\prime} \cap F_{i}\right| \geq t$ and $\left|F^{\prime} \cap F_{i} \cap M\right|=t-1$. Suppose $X=\left\{x_{1}, \ldots, x_{t}\right\}$. By the choice of $F_{1}, F_{2}, \ldots, F_{c-k}$, it is clear that $x_{1}, \ldots, x_{t}, y_{1} \ldots, y_{c-k}$ are in $F^{\prime}$.

Suppose that $c \geq 2 k-t+1$. If there exists $F^{\prime} \in \mathcal{F}$ such that $F^{\prime} \cap M=X$, by above discussion, then $\left|F^{\prime}\right|>k$. That is impossible. Hence $\left|F^{\prime \prime} \cap M\right| \geq t+1$ for any $F^{\prime \prime} \in \mathcal{F}_{X}$. By the maximality of $\mathcal{F}$, it is easy to see that any $k$-subset $F^{\prime \prime \prime}$ of $[n]$ satisfying $\left|F^{\prime \prime \prime} \cap X\right|=t-1$ and $\left|F^{\prime \prime \prime} \cap M\right|=k-1$ is in $\mathcal{F}$. Then we have $C=[n]$ and $c=n$. On the other hand, we have $c \geq k+2$, for otherwise we would have $c=k+1$, and $|T \cap F| \geq t$ for any $F \in \mathcal{F}$ and any $T \in\left(\begin{array}{c}C \\ t+1\end{array}\right)$ with $X \subseteq T$, which imply that $T \subseteq M$, a contradiction.

So far we have proved that either $k+2 \leq c \leq 2 k-t$ or $c=n$. It remains to prove (i) and (ii).

(i) Suppose that $k+2 \leq c \leq 2 k-t$. Since $|F \cap X|=t-1$ and $|F \cap M|=k-1$ for any $F \in \mathcal{F} \backslash \mathcal{F}_{X}$, we have $\mathcal{F} \backslash \mathcal{F}_{X} \subseteq \mathcal{C}(X, M, C)$. For any $F^{\prime} \in \mathcal{F}_{X}$, if $\left|F^{\prime} \cap M\right| \geq t+1$, then $F^{\prime} \in \mathcal{A}(X, M)$; if $F^{\prime} \cap M=X$, then $\left|F^{\prime} \cap C\right|=c-k+t$ by above discussion, which implies that $F^{\prime} \in \mathcal{B}(X, M, C)$. Thus, $\mathcal{F} \subseteq \mathcal{H}_{1}(X, M, C)$. By the maximality of $\mathcal{F}$, we get $\mathcal{F}=\mathcal{H}_{1}(X, M, C)$.

(ii) Suppose that $c=n$. Then $\mathcal{F}=\mathcal{A}(X, M) \cup \mathcal{C}(X, M, C)$ by the discussion in (i) and maximality of $\mathcal{F}$. If $t=k-2$, then $\mathcal{F}=\mathcal{H}_{1}(X, M,[n])=\mathcal{H}_{2}(M)$, which implies that $\mathcal{T}=\left(\begin{array}{c}M \\ t+1\end{array}\right)$ and $\tau_{t}(\mathcal{T})=t+1$, a contradiction.

Lemma 2.4 Let $n, k, t, \mathcal{F}$ and $\mathcal{T}$ be as in Assumption 1. Suppose $\tau_{t}(\mathcal{T})=t+1$ and $\mathcal{T}=\left(\begin{array}{c}Z \\ t+1\end{array}\right)$ for some $(t+2)$-subset $Z$ of $[n]$. Then $\mathcal{F}=\mathcal{H}_{2}(Z)$.

Proof. Since $\mathcal{T}=\left(\begin{array}{c}Z \\ t+1\end{array}\right)$, we have $|F \cap Z| \geq t$ for any $F \in \mathcal{F}$. If there exists $F^{\prime} \in \mathcal{F}$ such that $\left|F^{\prime} \cap Z\right|=t$, then there exists a $T^{\prime} \in \mathcal{T}$ such that $\left|F^{\prime} \cap T^{\prime}\right|=t-1$, a contradiction. Hence, $\mathcal{F} \subseteq \mathcal{H}_{2}(Z)$. Since $\mathcal{F}$ is maximal and $\mathcal{H}_{2}(Z)$ is $t$-intersecting, we have $\mathcal{F}=\mathcal{H}_{2}(Z)$.

Now we give some equalities and inequalities for the sizes of Families I and II. 
Lemma 2.5 Suppose $c \in\{k+1, k+2, \ldots, 2 k-t, n\}$. Then the following hold.

$$
\begin{aligned}
& h_{1}(t, k, c)=\left(\begin{array}{l}
n-t \\
k-t
\end{array}\right)-\left(\begin{array}{l}
n-k \\
k-t
\end{array}\right)+\left(\begin{array}{c}
n-c \\
2 k-c-t
\end{array}\right)+t(c-k) . \\
& h_{2}(t+2)=(t+2)\left(\begin{array}{l}
n-t-2 \\
k-t-1
\end{array}\right)+\left(\begin{array}{l}
n-t-2 \\
k-t-2
\end{array}\right) .
\end{aligned}
$$

Proof. Let $X, M, C, \mathcal{A}(X, M), \mathcal{B}(X, M, C)$ and $\mathcal{C}(X, M, C)$ be as in Family I. Then

$$
|\mathcal{A}(X, M)|=\left(\begin{array}{l}
n-t \\
k-t
\end{array}\right)-\left(\begin{array}{l}
n-k \\
k-t
\end{array}\right),|\mathcal{B}(X, M, C)|=\left(\begin{array}{c}
n-c \\
2 k-c-t
\end{array}\right),|\mathcal{C}(X, M, C)|=t(c-k) .
$$

Hence, (2) holds.

Consider the family $\mathcal{H}_{2}(Z)$, where $Z$ is a $(t+2)$-subset of $[n]$. Observe that the number of $k$-subsets $F$ of $[n]$ satisfying $|F \cap Z|=t+1$ is $(t+2)\left(\begin{array}{c}n-t-2 \\ k-t-1\end{array}\right)$, and the number of $k$-subsets $F$ of $[n]$ satisfying $|F \cap Z|=t+2$ is $\left(\begin{array}{c}n-t-2 \\ k-t-2\end{array}\right)$. Hence we have (3) holds.

Let

$$
f(n, k, t)=(k-t)\left(\begin{array}{c}
n-t-1 \\
k-t-1
\end{array}\right)-\left(\begin{array}{c}
k-t \\
2
\end{array}\right)\left(\begin{array}{l}
n-t-2 \\
k-t-2
\end{array}\right)
$$

Lemma 2.6 Let $1 \leq t \leq k-2$ and $2 k<n$. Then the following hold.

(i) $h_{1}(t, k, k+1)>h_{1}(t, k, k+2)>\cdots>h_{1}(t, k, 2 k-t)$.

(ii) $\min \left\{h_{1}(t, k, 2 k-t), h_{1}(t, k, n)\right\} \geq f(n, k, t)$.

Proof. (i) For any $c \in\{k+1, k+2, \ldots, 2 k-t-1\}$, we have

$$
h_{2}(t, k, c)-h_{2}(t, k, c+1)=\left(\begin{array}{c}
n-c-1 \\
2 k-c-t
\end{array}\right)-t=\prod_{i=0}^{2 k-c-t-1} \frac{n-c-1-i}{2 k-c-t-i}-t .
$$

Observe that $\frac{n-c-1-i}{2 k-c-t-i}>1$ for any $i \in\{0,1, \ldots, 2 k-c-t-1\}$, and $\frac{n-c-1-i}{2 k-c-t-i}=n-2 k+t>t$ when $i=2 k-c-t-1$. Hence, we have $h_{2}(t, k, c)>h_{2}(t, k, c+1)$ for any $c \in\{k+1, k+$ $2, \ldots, 2 k-t-1\}$, and (i) holds.

(ii) Let $X$ and $M$ be as in Family I. For any $i \in\{t, t+1, \ldots, k\}$, denote $\mathcal{A}_{i}(X, M)=$ $\{F \subseteq[n]|X \subseteq F| F,|=k|, F \cap M \mid=i\}$ and

$$
\mathcal{L}_{i}(X, M)=\left\{(I, F) \in\left(\begin{array}{c}
{[n]} \\
i
\end{array}\right) \times\left(\begin{array}{c}
{[n]} \\
k
\end{array}\right) \mid X \subseteq I \subseteq M, I \subseteq F\right\} .
$$

Double counting $\left|\mathcal{L}_{i}(X, M)\right|$, we obtain

$$
\left|\mathcal{L}_{i}(X, M)\right|=\sum_{j=i}^{k}\left|\mathcal{A}_{j}(X, M)\right| \cdot\left(\begin{array}{c}
j-t \\
i-t
\end{array}\right)=\left(\begin{array}{c}
k-t \\
i-t
\end{array}\right)\left(\begin{array}{l}
n-i \\
k-i
\end{array}\right) .
$$

Since $\mathcal{A}(X, M)=\cup_{j=t+1}^{k} \mathcal{A}_{j}(X, M)$ and

$$
\left|\mathcal{L}_{t+1}(X, M)\right|=\sum_{j=t+1}^{k}\left|\mathcal{A}_{j}(X, M)\right|+\sum_{j=t+2}^{k}\left|\mathcal{A}_{j}(X, M)\right| \cdot(j-t-1),
$$


we obtain

$$
\begin{aligned}
\left|\mathcal{L}_{t+1}(X, M)\right| & =(k-t)\left(\begin{array}{l}
n-t-1 \\
k-t-1
\end{array}\right) \leq|\mathcal{A}|+\sum_{j=t+2}^{k}\left|\mathcal{A}_{j}(X, M)\right| \cdot\left(\begin{array}{c}
j-t \\
2
\end{array}\right) \\
& =|\mathcal{A}|+\left|\mathcal{L}_{t+2}(X, M)\right|=|\mathcal{A}|+\left(\begin{array}{c}
k-t \\
2
\end{array}\right)\left(\begin{array}{c}
n-t-2 \\
k-t-2
\end{array}\right)
\end{aligned}
$$

which implies that $|\mathcal{A}(X, M)| \geq f(n, k, t)$. From the construction of $\mathcal{H}_{1}(X, M, C)$, we have (ii) holds.

Lemma 2.7 Let $1 \leq t \leq k-2$ and $\left(\begin{array}{c}t+2 \\ 2\end{array}\right)(k-t+1)^{2}+t \leq n$. Then the following hold.

(i) If $1 \leq t \leq \frac{k}{2}-\frac{3}{2}$, then $h_{2}(t+2)<f(n, k, t)$.

(ii) If $\frac{k}{2}-\frac{3}{2}<t \leq k-2$, then $h_{2}(t+2)>f(n, k, t)$.

Proof. Note that

$$
h_{2}(t+2)=(t+2)\left(\begin{array}{l}
n-t-1 \\
k-t-1
\end{array}\right)-(t+1)\left(\begin{array}{l}
n-t-2 \\
k-t-2
\end{array}\right) .
$$

Let $f_{2}(n, k, t)=\left(f(n, k, t)-h_{2}(t+2)\right) /\left(\begin{array}{c}n-t-2 \\ k-t-2\end{array}\right)$. Then

$$
f_{2}(n, k, t)=\frac{(k-2 t-2)(n-t-1)}{k-t-1}-\left(\begin{array}{c}
k-t \\
2
\end{array}\right)+t+1
$$

(i) From $1 \leq t \leq \frac{k}{2}-\frac{3}{2}$ and $\left(\begin{array}{c}t+2 \\ 2\end{array}\right)(k-t+1)^{2}+t \leq n$, we have

$$
f_{2}(n, k, t) \geq \frac{(k-2 t-2)(t+2)(t+1)(k-t+1)^{2}}{2(k-t-1)}-\frac{(k-t)(k-t-1)}{2}+t+\frac{t+1}{k-t-1} .
$$

Since $(k-2 t-2)(t+2)(t+1) \geq(k-2 t-2)+(t+2)=k-t$, we have $f_{2}(n, k, t)>0$ and (i) holds.

(ii) If $t=\frac{k}{2}-1$, then $f_{2}(n, k, t)=-t(t+1) / 2<0$. If $\frac{k}{2}-\frac{1}{2} \leq t \leq k-2$, then $k-2 t-2<0$ and

$$
\begin{aligned}
f_{2}(n, k, t) & =\frac{(k-2 t-2) n+(t+1)^{2}}{k-t-1}-\left(\begin{array}{c}
k-t \\
2
\end{array}\right) \\
& \leq \frac{(k-2 t-2)(t+2)(t+1)(k-t+1)^{2}+2(t+1)^{2}}{2(k-t-1)}+\frac{(k-2 t-2) t}{k-t-1}-\left(\begin{array}{c}
k-t \\
2
\end{array}\right)<0 .
\end{aligned}
$$

Therefore, we have (ii) holds.

Lemma 2.8 Let $1 \leq t \leq k-2$ and $\left(\begin{array}{c}t+2 \\ 2\end{array}\right)(k-t+1)^{2}+t \leq n$. Then the following hold.

(i) Suppose that $1 \leq t \leq k-3$. Then $h_{1}(t, k, 2 k-t-2)>h_{1}(t, k, n) \geq h_{1}(t, k, 2 k-t-1)$, and equality holds only if $t=1$. 
(ii) Suppose that $t=k-2$. Then $h_{1}(t, k, n) \geq h_{1}(t, k, k+1)$, and equality holds only if $t=1$.

Proof. From Lemma 2.5, we have

$$
\begin{aligned}
& h_{1}(t, k, 2 k-t-2)-h_{1}(t, k, n)=\frac{1}{2}(n-2 k+t+2)(n-2 k-t+1), \\
& h_{1}(t, k, n)-h_{1}(t, k, 2 k-t-1)=(t-1)(n-2 k+t+1) .
\end{aligned}
$$

Then (i) holds from

$$
n-2 k-t \geq\left(\begin{array}{c}
t+2 \\
2
\end{array}\right)(k-t+1)^{2}-2 k \geq 2(t+2)(k-t+1)-2 k=2(t+1)(k-t)+4>0 .
$$

If $t=k-2$, then $h_{1}(t, k, n)-h_{1}(t, k, k+1)=(n-k-1)(t-1)$ and (ii) holds.

Lemma 2.9 Let $1 \leq t \leq k-2$ and $2 k<n$. Then the following hold.

(i) Suppose that $t=\frac{k}{2}-1$. Then $h_{2}(t+2) \geq h_{1}(t, k, k+2)$ and equality holds only if $t=1$; if $t=1$, or $t \geq 2$ and $n$ is sufficiently large, then $h_{1}(t, k, k+1)>h_{2}(t+2)$.

(ii) Suppose that $\frac{k}{2}-\frac{1}{2} \leq t \leq k-2$. Then $h_{2}(t+2) \geq h_{1}(t, k, k+1)$ and equality holds only if $(t, k)=(1,3)$.

Proof. Since $\left(\begin{array}{c}n-t \\ k-t\end{array}\right)=\sum_{i=0}^{k-t-1}\left(\begin{array}{c}n-k+i \\ k-t-1\end{array}\right)+\left(\begin{array}{c}n-k \\ k-t\end{array}\right)$, we have

$$
h_{1}(t, k, c)=\sum_{i=0}^{k-t-1}\left(\begin{array}{c}
n-k+i \\
k-t-1
\end{array}\right)+\left(\begin{array}{c}
n-c \\
2 k-c-t
\end{array}\right)+t(c-k)
$$

for any $c \in\{k+1, k+2, \ldots, 2 k-t, n\}$. By (4) and (5), we have

$$
\begin{aligned}
& h_{2}(t+2)-h_{1}(t, k, k+1) \\
= & (t+1)\left(\left(\begin{array}{l}
n-t-1 \\
k-t-1
\end{array}\right)-\left(\begin{array}{l}
n-t-2 \\
k-t-2
\end{array}\right)\right)-\sum_{i=-1}^{k-t-2}\left(\begin{array}{l}
n-k+i \\
k-t-1
\end{array}\right)-t \\
= & (t+1)\left(\begin{array}{l}
n-t-2 \\
k-t-1
\end{array}\right)-\sum_{i=-1}^{k-t-2}\left(\begin{array}{l}
n-k+i \\
k-t-1
\end{array}\right)-t \\
= & (2 t+1-k)\left(\begin{array}{l}
n-t-2 \\
k-t-1
\end{array}\right)+\sum_{i=-1}^{k-t-3}\left(\left(\begin{array}{l}
n-t-2 \\
k-t-1
\end{array}\right)-\left(\begin{array}{l}
n-k+i \\
k-t-1
\end{array}\right)\right)-t .
\end{aligned}
$$

If $\frac{k}{2}-\frac{1}{2} \leq t \leq k-3$, then $h_{2}(t+2)-h_{1}(t, k, k+1) \geq\left(\begin{array}{c}n-t-2 \\ k-t-1\end{array}\right)-\left(\begin{array}{c}n-k-1 \\ k-t-1\end{array}\right)-t>0$. If $1=t=k-2$, then $h_{2}(t+2)-h_{1}(t, k, k+1)=0$. If $2 \leq t=k-2$, then $h_{2}(t+2)-h_{1}(t, k, k+1)=$ $(t-1)(n-t-2)+1-t>0$.

Suppose that $\frac{k}{2}-1=t$. If $t=1$, then $h_{2}(t+2)-h_{1}(t, k, k+1)=\frac{1}{2}(n-5)(-n+8)<0$. When $t \geq 2$, note that $h_{2}(t+2)-h_{1}(t, k, k+1)$ is a polynomial in $n$ with negative leading 
coefficient. Then $h_{2}(t+2)-h_{1}(t, k, k+1)<0$ if $t \geq 2$ and $n$ is sufficiently large. By (4) and (5) again, we have

$$
h_{2}(t+2)-h_{1}(t, k, k+2)=t\left(\begin{array}{c}
n-t-2 \\
t+1
\end{array}\right)-\sum_{i=0}^{t-1}\left(\begin{array}{c}
n-2 t-2+i \\
t+1
\end{array}\right)-\left(\begin{array}{c}
n-2 t-4 \\
t
\end{array}\right)-2 t .
$$

If $t=1$, then $h_{2}(t+2)-h_{1}(t, k, k+2)=0$. If $t \geq 2$, then

$$
h_{2}(t+2)-h_{1}(t, k, k+2)>\left(\begin{array}{c}
n-t-2 \\
t+1
\end{array}\right)-\left(\begin{array}{c}
n-2 t-2 \\
t+1
\end{array}\right)-\left(\begin{array}{c}
n-2 t-4 \\
t
\end{array}\right)-2 t>0
$$

from $\left(\begin{array}{c}n-t-3 \\ t\end{array}\right)>n-2 t-2>2 t$ and $\left(\begin{array}{c}n-t-2 \\ t+1\end{array}\right)=\left(\begin{array}{c}n-t-4 \\ t+1\end{array}\right)+\left(\begin{array}{c}n-t-4 \\ t\end{array}\right)+\left(\begin{array}{c}n-t-3 \\ t\end{array}\right)$. Hence, the desired result follows.

\section{$3 \quad$ Upper bounds for non-trivial $t$-intersecting families}

In [6], Frankl also proved that the size of any $t$-intersecting family $\mathcal{F}$ is no more than $c_{k}^{\prime}\left(\begin{array}{c}n-\ell_{1} \\ k-\ell_{1}\end{array}\right)$, where $\ell_{1}$ is the minimum size of a subset in the base of $\mathcal{F}$ and $c_{k}^{\prime}$ is a constant depending only on $k$. In this section, we give some specifical upper bounds on the sizes of the maximal non-trivial $t$-intersecting families. For any family $\mathcal{F} \subseteq\left(\begin{array}{c}{[n]} \\ k\end{array}\right)$ and any subset $S$ of $[n]$, define $\mathcal{F}_{S}=\{F \in \mathcal{F} \mid S \subseteq F\}$.

Lemma 3.1 Let $\mathcal{F} \subseteq\left(\begin{array}{c}{[n]} \\ k\end{array}\right)$ be a t-intersecting family and $S$ an s-subset of $[n]$, where $t-1 \leq$ $s \leq k-1$. If there exists $F^{\prime} \in \mathcal{F}$ such that $\left|S \cap F^{\prime}\right|=r \leq t-1$, then for each $i \in\{1,2, \ldots, t-r\}$ there exists an $(s+i)$-subset $T_{i}$ with $S \subseteq T_{i}$ such that $\left|\mathcal{F}_{S}\right| \leq\left(\begin{array}{c}k-r \\ i\end{array}\right)\left|\mathcal{F}_{T_{i}}\right|$.

Proof. For any $i \in\{1,2, \ldots, t-r\}$, let

$$
\mathcal{H}_{i}=\left\{H \in\left(\begin{array}{c}
S \cup F^{\prime} \\
s+i
\end{array}\right) \mid S \subseteq H\right\}
$$

Observe that $\left|\mathcal{H}_{i}\right|=\left(\begin{array}{c}k-r \\ i\end{array}\right)$. For any $F \in \mathcal{F}_{S}$, since $\mathcal{F}$ is $t$-intersecting, we have $\left|F \cap F^{\prime}\right| \geq t$, implying that $\left|F \cap\left(S \cup F^{\prime}\right)\right| \geq s+t-r$ and there exists $H \in \mathcal{H}_{i}$ such that $H \subseteq F$. Therefore $\mathcal{F}_{S}=\cup_{H \in \mathcal{H}_{i}} \mathcal{F}_{H}$. Let $T_{i}$ be a subset in $\mathcal{H}_{i}$ such that $\left|\mathcal{F}_{H}\right| \leq\left|\mathcal{F}_{T_{i}}\right|$ for any $\bar{H} \in \mathcal{H}_{i}$. Thus $\left|\mathcal{F}_{S}\right| \leq\left(\begin{array}{c}k-r \\ i\end{array}\right)\left|\mathcal{F}_{T_{i}}\right|$ as desired.

Since $\left|\mathcal{F}_{T}\right| \leq\left(\begin{array}{c}n-|T| \\ k-|T|\end{array}\right)$ for any subset $T$ of $[n]$, we can obtain the following lemma.

Lemma 3.2 Let $\mathcal{F} \subseteq\left(\begin{array}{c}{[n]} \\ k\end{array}\right)$ be a t-intersecting family and $S$ an s-subset of $[n]$ with $t-1 \leq$ $s \leq k$. If there exists $F^{\prime} \in \mathcal{F}$ such that $\operatorname{dim}\left(S \cap F^{\prime}\right)=r \leq t-1$, then $\left|\mathcal{F}_{S}\right| \leq\left(\begin{array}{c}k-r \\ t-r\end{array}\right)\left(\begin{array}{c}n-s-t+r \\ k-s-t+r\end{array}\right)$.

The following lemma gives some upper bounds on the size of maximal non-trivial $t$ intersecting families $\mathcal{F}$ with $\tau_{t}(\mathcal{F})=t+1$.

Lemma 3.3 Let $n, k, t, \mathcal{F}$ and $\mathcal{T}$ be as in Assumption 1. Then the following hold.

(i) If $|\mathcal{T}|=1$, then $|\mathcal{F}| \leq\left(\begin{array}{c}n-t-1 \\ k-t-1\end{array}\right)+(t+1)(k-t)(k-t+1)\left(\begin{array}{c}n-t-2 \\ k-t-2\end{array}\right)$. 
(ii) Suppose that $|\mathcal{T}| \geq 2$ and $\mathcal{T}=\left\{T \in\left(\begin{array}{c}M \\ t+1\end{array}\right) \mid X \subseteq T\right\}$ for some $t$-subset $X$ and l-subset $M$ of $[n]$ with $X \subseteq M$. Then

$$
|\mathcal{F}| \leq(l-t)\left(\begin{array}{c}
n-t-1 \\
k-t-1
\end{array}\right)+(k-l+1)(k-t+1)\left(\begin{array}{c}
n-t-2 \\
k-t-2
\end{array}\right)+t\left(\begin{array}{c}
n-l \\
k-l+1
\end{array}\right) .
$$

Moreover, if $l=t+2$, then

$$
|\mathcal{F}| \leq 2\left(\begin{array}{l}
n-t-1 \\
k-t-1
\end{array}\right)+(k-1)(k-t+1)\left(\begin{array}{l}
n-t-2 \\
k-t-2
\end{array}\right) .
$$

(iii) If $|\mathcal{T}| \geq 2$ and $\mathcal{T}=\left(\begin{array}{c}Z \\ t+1\end{array}\right)$ for some $(t+2)$-subset $Z$ of $[n]$, then $|\mathcal{F}|=h_{2}(t+2)$.

Proof. (i) Let $T$ be the unique element in $\mathcal{T}$. Since $|T \cap F| \geq t$ for any $F \in \mathcal{F}$, we have

$$
\mathcal{F}=\mathcal{F}_{T} \cup\left(\bigcup_{S \in\left(\begin{array}{c}
T \\
t
\end{array}\right)}\left(\mathcal{F}_{S} \backslash \mathcal{F}_{T}\right)\right)
$$

We now give an upper bound on $\left|\mathcal{F}_{S} \backslash \mathcal{F}_{T}\right|$ for any fixed $S \in\left(\begin{array}{c}T \\ t\end{array}\right)$. Since $\tau_{t}(\mathcal{F})=t+1$, there exists an $F^{\prime} \in \mathcal{F} \backslash \mathcal{F}_{S}$ such that $\left|S \cap F^{\prime}\right|=t-1$ from $\left|F^{\prime} \cap T\right| \geq t$. Then we have $T=\left(F^{\prime} \cap T\right) \cup S$ and $T \subseteq F^{\prime} \cup S$. For any $F \in \mathcal{F}_{S} \backslash \mathcal{F}_{T}$, notice that $\left(F \cap F^{\prime}\right) \cup S \subseteq F \cap\left(F^{\prime} \cup S\right)$. Since $\left|F \cap F^{\prime}\right| \geq t$ and $\left|F \cap F^{\prime} \cap S\right| \leq t-1$, we have $\left|F \cap\left(F^{\prime} \cup S\right)\right| \geq t+1$. Hence there exists a $(t+1)$-subset $H$ such that $H \neq T, S \subseteq H \subseteq S \cup F^{\prime}$ and $H \subseteq F$. Therefore, we have

$$
\mathcal{F}_{S} \backslash \mathcal{F}_{T}=\bigcup_{\substack{S \subseteq H \subseteq S \cup F^{\prime} \\ H \neq T,|H|=t+1}} \mathcal{F}_{H}
$$

Consider any $(t+1)$-subset $H$ of $[n]$ satisfying $H \neq T$ and $S \subseteq H \subseteq S \cup F^{\prime}$. Since $T$ is the unique $(t+1)$-subset of $[n]$ such that $|T \cap F| \geq t$ for any $F \in \mathcal{F}$, there exists $F^{\prime \prime}$ such that $\left|H \cap F^{\prime \prime}\right|<t$, which implies that $\left|H \cap F^{\prime \prime}\right|=t-1$ from $|H \cap T|=|S|=t$ and $\left|T \cap F^{\prime \prime}\right| \geq t$. From Lemma 3.2, we have $\left|\mathcal{F}_{H}\right| \leq(k-t+1)\left(\begin{array}{c}n-t-2 \\ k-t-2\end{array}\right)$. Observe $\left|\mathcal{F}_{T}\right| \leq\left(\begin{array}{l}n-t-1 \\ k-t-1\end{array}\right)$ and

$$
\left|\left\{H \in\left(\begin{array}{c}
S \cup F^{\prime} \\
t+1
\end{array}\right) \mid S \subseteq H, H \neq T\right\}\right|=k-t .
$$

Therefore, from (8) and (9), we obtain

$$
|\mathcal{F}| \leq\left(\begin{array}{l}
n-t-1 \\
k-t-1
\end{array}\right)+(t+1)(k-t)(k-t+1)\left(\begin{array}{c}
n-t-2 \\
k-t-2
\end{array}\right),
$$

as desired.

(ii) We will obtain the upper bound of $|\mathcal{F}|$ by establishing upper bounds on $\left|\mathcal{F}_{X}\right|$ and $\left|\mathcal{F} \backslash \mathcal{F}_{X}\right|$. Since $\tau_{t}(\mathcal{F})=t+1$, we have $|F \cap X| \geq t-1$ for any $F \in \mathcal{F}$, and there exists $F^{\prime} \in \mathcal{F}$ such that $\left|X \cap F^{\prime}\right|=t-1$. From the proof of Lemma 2.1, we have $X \subseteq M \subseteq X \cup F^{\prime}$.

For any $F \in \mathcal{F}_{X}$, we have $\left|F \cap\left(X \cup F^{\prime}\right)\right| \geq t+1$ from $X \subseteq F$ and $\left|F \cap F^{\prime}\right| \geq t$. So

$$
\mathcal{F}_{X}=\left(\bigcup_{X \subseteq H_{1}, H_{1} \in\left(\begin{array}{c}
M \\
t+1
\end{array}\right)} \mathcal{F}_{H_{1}}\right) \cup\left(\bigcup_{X \subseteq H_{2}, H_{2} \in\left(\begin{array}{c}
X \cup F^{\prime} \\
t+1
\end{array}\right) \backslash\left(\begin{array}{c}
M \\
t+1
\end{array}\right)} \mathcal{F}_{H_{2}}\right) .
$$


Since $\left|\mathcal{F}_{H_{1}}\right| \leq\left(\begin{array}{c}n-(t+1) \\ k-(t+1)\end{array}\right)$ for any $H_{1} \in\left(\begin{array}{c}M \\ t+1\end{array}\right)$, we have $\left|\bigcup_{X \subseteq H_{1}, H_{1} \in\left(\begin{array}{c}M \\ t+1\end{array}\right)} \mathcal{F}_{H_{1}}\right| \leq(l-t)\left(\begin{array}{c}n-(t+1) \\ k-(t+1)\end{array}\right)$. For any $H_{2} \in\left(\begin{array}{c}X \cup F^{\prime} \\ t+1\end{array}\right) \backslash\left(\begin{array}{c}M \\ t+1\end{array}\right)$ with $X \subseteq H_{2}$, since $H_{2} \notin \mathcal{T}$, there exists $F^{\prime \prime} \in \mathcal{F}$ such that $\left|H_{2} \cap F^{\prime \prime}\right|<t$, which implies that $\left|H_{2} \cap F^{\prime \prime}\right|=t-1$ from $\left|F^{\prime \prime} \cap X\right| \geq t-1$. It follows that $\left|\mathcal{F}_{H_{2}}\right| \leq(k-t+1)\left(\begin{array}{c}n-(t+1)-1 \\ k-(t+1)-1\end{array}\right)$ from Lemma 3.2. Notice that

$$
\left|\left\{H_{2} \in\left(\begin{array}{c}
X \cup F^{\prime} \\
t+1
\end{array}\right) \backslash\left(\begin{array}{c}
M \\
t+1
\end{array}\right) \mid X \subseteq H_{2}\right\}\right|=k-l+1 .
$$

Therefore, we have

$$
\left|\mathcal{F}_{X}\right| \leq(l-t)\left(\begin{array}{l}
n-t-1 \\
k-t-1
\end{array}\right)+(k-l+1)(k-t+1)\left(\begin{array}{l}
n-t-2 \\
k-t-2
\end{array}\right) .
$$

For any $F \in \mathcal{F} \backslash \mathcal{F}_{X}$ and any $T \in \mathcal{T}$, since $|F \cap X|=t-1$ and $X \nsubseteq F \cap T$, we have $T=(F \cap T) \cup X \subseteq F \cup X$. Then for any $F \in \mathcal{F} \backslash \mathcal{F}_{X}$ we have $M=\cup_{T \in \mathcal{T}} T \subseteq F \cup X$, which implies that $|M \cap F|=l-1$. Hence, $\mathcal{F} \backslash \mathcal{F}_{X} \subseteq\left\{F \in\left(\begin{array}{c}{[n]} \\ k\end{array}\right)|| F \cap M \mid=l-1, X \nsubseteq F\right\}$, and

$$
\left|\mathcal{F} \backslash \mathcal{F}_{X}\right| \leq t\left(\begin{array}{c}
n-l \\
k-l+1
\end{array}\right)
$$

Combining (11) and (12), we obtain (6).

Now let us consider the case when $l=t+2$. From the discussion above, we have $|M \cap F|=l-1=t+1$ for any $F \in \mathcal{F} \backslash \mathcal{F}_{X}$, which implies that

$$
\mathcal{F} \backslash \mathcal{F}_{X} \subseteq \bigcup_{X \nsubseteq L, L \in\left(\begin{array}{c}
M \\
t+1
\end{array}\right)} \mathcal{F}_{L} .
$$

For any $L \in\left(\begin{array}{c}M \\ t+1\end{array}\right)$ with $X \nsubseteq L$, since $L \notin \mathcal{T}$ and $|F \cap M| \geq t$ for any $F \in \mathcal{F}$, there exists $F^{\prime} \in \mathcal{F}$ such that $\left|F^{\prime} \cap L\right|=t-1$. Then $\left|\mathcal{F}_{L}\right| \leq(k-t+1)\left(\begin{array}{l}n-t-2 \\ k-t-2\end{array}\right)$ from Lemma 3.2. Since the number of $(t+1)$-subsets $L$ of $M$ with $X \nsubseteq L$ is equal to $t$, we have

$$
\left|\mathcal{F} \backslash \mathcal{F}_{X}\right| \leq t(k-t+1)\left(\begin{array}{l}
n-t-2 \\
k-t-2
\end{array}\right)
$$

Combining (11) and (13), we obtain (7).

(iii) By Lemma 2.4, the desired result follows.

Lemma 3.4 Let $n, k$ and $t$ be integers with $1 \leq t \leq k-2$ and $\left(\begin{array}{c}t+2 \\ 2\end{array}\right)(k-t+1)^{2}+t \leq n$, and let $\mathcal{F} \subseteq\left(\begin{array}{c}{[n]} \\ k\end{array}\right)$ be a maximal $t$-intersecting family with $t+2 \leq \tau_{t}(\mathcal{F})=m \leq k$. Then

$$
|\mathcal{F}| \leq k^{m-t-2}(k-t+1)^{2}\left(\begin{array}{c}
m \\
t
\end{array}\right)\left(\begin{array}{c}
n-m \\
k-m
\end{array}\right) \text {. }
$$

Moreover, we have

$$
|\mathcal{F}| \leq(k-t+1)^{2}\left(\begin{array}{c}
t+2 \\
2
\end{array}\right)\left(\begin{array}{l}
n-t-2 \\
k-t-2
\end{array}\right)
$$


Proof. Let $T$ be an $m$-subset of $[n]$ which satisfies $|T \cap F| \geq t$ for any $F \in \mathcal{F}$. Then $\mathcal{F}=\cup_{H \in\left(\begin{array}{c}T \\ t\end{array}\right)} \mathcal{F}_{H}$ and there exists $H_{1} \in\left(\begin{array}{c}T \\ t\end{array}\right)$ such that $|\mathcal{F}| \leq\left(\begin{array}{c}m \\ t\end{array}\right)\left|\mathcal{F}_{H_{1}}\right|$. If $m \geq t+3$, using Lemma 3.1 repeatedly, then there exist $H_{2} \in\left(\begin{array}{c}{[n]} \\ t+1\end{array}\right), H_{3} \in\left(\begin{array}{c}{[n]} \\ t+2\end{array}\right), \ldots, H_{m-t-1} \in\left(\begin{array}{c}{[n]} \\ m-2\end{array}\right)$ such that $H_{i} \subseteq H_{i+1}$ and $\left|\mathcal{F}_{H_{i}}\right| \leq k\left|\mathcal{F}_{H_{i+1}}\right|$ for each $i \in\{1,2, \ldots, m-t-2\}$. Thus there exists $H^{\prime} \in\left(\begin{array}{c}{[n]} \\ m-2\end{array}\right)$ such that

$$
|\mathcal{F}| \leq\left(\begin{array}{c}
m \\
t
\end{array}\right) k^{m-t-2}\left|\mathcal{F}_{H^{\prime}}\right|
$$

Since $\tau_{t}(\mathcal{F})>m-2$, we have $\mathcal{F} \backslash \mathcal{F}_{H^{\prime}} \neq \emptyset$ and $\left|F \cap H^{\prime}\right| \leq t-1$ for any $F \in \mathcal{F} \backslash \mathcal{F}_{H^{\prime}}$.

Case 1. $\left|F \cap H^{\prime}\right| \leq t-2$ for all $F \in \mathcal{F} \backslash \mathcal{F}_{H^{\prime}}$.

In this case, we have $t \geq 2$. For $s \in\{0,1, \ldots, t-2\}$, let

$$
g(s)=\left(\begin{array}{c}
k-s \\
t-s
\end{array}\right)\left(\begin{array}{l}
n-m+2-t+s \\
k-m+2-t+s
\end{array}\right)
$$

Since $t+2 \leq m, s \leq t-2$ and $\left(\begin{array}{c}t+2 \\ 2\end{array}\right)(k-t+1)^{2}+t \leq n$, we have

$$
\begin{aligned}
& (t-s)(n-m+3-t+s)-(k-s)(k-m+3-t+s) \\
= & (k-t) m+(t-s)(n-k)-(k-t)(k+3-t+s) \\
> & (k-t)(t+2)+n-k-(k-t)(k+1) \\
= & n-t-(k-t)^{2}>0,
\end{aligned}
$$

which implies that

$$
\frac{g(s+1)}{g(s)}=\frac{(t-s)(n-m+3-t+s)}{(k-s)(k-m+3-t+s)}>1
$$

for $s \in\{0,1, \ldots, t-3\}$. That is the function $g(s)$ is increasing as $s \in\{0,1, \ldots, t-2\}$ increases.

Let $F_{1}$ be a fixed $k$-subset in $\mathcal{F} \backslash \mathcal{F}_{H^{\prime}}$. Assume that $\left|F_{1} \cap H^{\prime}\right|=s_{1}$. Observe that $0 \leq s_{1} \leq t-2$. By Lemma 3.2, we have $\left|\mathcal{F}_{H^{\prime}}\right| \leq g\left(s_{1}\right) \leq g(t-2)$, which implies that

$$
|\mathcal{F}| \leq\left(\begin{array}{c}
m \\
t
\end{array}\right) k^{m-t-2} g(t-2)=k^{m-t-2}\left(\begin{array}{c}
m \\
t
\end{array}\right)\left(\begin{array}{c}
k-t+2 \\
2
\end{array}\right)\left(\begin{array}{c}
n-m \\
k-m
\end{array}\right) .
$$

Case 2. There exists $F_{2} \in \mathcal{F} \backslash \mathcal{F}_{H^{\prime}}$ such that $\left|F_{2} \cap H^{\prime}\right|=t-1$.

By Lemma 3.1, there exists an $(m-1)$-subset $H^{\prime \prime}$ such that $\left|\mathcal{F}_{H^{\prime}}\right| \leq(k-t+1)\left|\mathcal{F}_{H^{\prime \prime}}\right|$. Hence, we have $|\mathcal{F}| \leq\left(\begin{array}{c}m \\ t\end{array}\right) k^{m-t-2}(k-t+1)\left|\mathcal{F}_{H^{\prime \prime}}\right|$. Since $\tau_{t}(\mathcal{F})>m-1$, there exists $F_{3} \in \mathcal{F}$ such that $\left|F_{3} \cap H^{\prime \prime}\right| \leq t-1$.

If $\left|F_{3} \cap H^{\prime \prime}\right|=t-1$, then there exists an $m$-subset $H^{\prime \prime \prime}$ of $[n]$ with $H^{\prime \prime} \subseteq H^{\prime \prime \prime}$ such that $\left|\mathcal{F}_{H^{\prime \prime}}\right| \leq(k-t+1)\left|\mathcal{F}_{H^{\prime \prime \prime}}\right|$. Since $\left|\mathcal{F}_{H^{\prime \prime \prime}}\right| \leq\left(\begin{array}{c}n-m \\ k-m\end{array}\right)$, we have

$$
|\mathcal{F}| \leq k^{m-t-2}(k-t+1)^{2}\left(\begin{array}{c}
m \\
t
\end{array}\right)\left(\begin{array}{c}
n-m \\
k-m
\end{array}\right) .
$$

Suppose that $t \geq 2$ and $\left|F_{3} \cap H^{\prime \prime}\right|=s_{2} \leq t-2$. By Lemma 3.2, we have

$$
\left|\mathcal{F}_{H^{\prime \prime}}\right| \leq\left(\begin{array}{c}
k-s_{2} \\
t-s_{2}
\end{array}\right)\left(\begin{array}{l}
n-m+1-t+s_{2} \\
k-m+1-t+s_{2}
\end{array}\right) \text {. }
$$


Similar to Case 1, it is straightforward to verify that the function $\left(\begin{array}{c}k-s \\ t-s\end{array}\right)\left(\begin{array}{c}n-m+1-t+s \\ k-m+1-t+s\end{array}\right)$ is increasing as $s \in\{0,1, \ldots, t-2\}$ increases. Hence

$$
|\mathcal{F}| \leq k^{m-t-2}(k-t+1)\left(\begin{array}{c}
m \\
t
\end{array}\right)\left(\begin{array}{c}
k-t+2 \\
2
\end{array}\right)\left(\begin{array}{l}
n-m-1 \\
k-m-1
\end{array}\right) .
$$

If $t=1$, then (14) holds from (17). If $t \geq 2$, from $\left(\begin{array}{c}t+2 \\ 2\end{array}\right)(k-t+1)^{2}+t \leq n$, it is straightforward to verify that

$(k-t+1)^{2}\left(\begin{array}{l}n-m \\ k-m\end{array}\right) \geq \max \left\{\left(\begin{array}{c}k-t+2 \\ 2\end{array}\right)\left(\begin{array}{l}n-m \\ k-m\end{array}\right),(k-t+1)\left(\begin{array}{c}k-t+2 \\ 2\end{array}\right)\left(\begin{array}{l}n-m-1 \\ k-m-1\end{array}\right)\right\}$,

which together with (16), (17) and (18) yields that (14) holds.

Let $p(x)=(x-t+1)(n-x)-k(x+1)(k-x)$ for each $x \in\{t+1, t+2, \ldots, k\}$. Observe that

$$
\begin{aligned}
p(t+1) & =2(n-t-1)-k(t+2)(k-t-1) \\
& \geq(t+2)(t+1)(k-t+1)^{2}-k(t+2)(k-t+1)+2 k(t+2)-2>0,
\end{aligned}
$$

and

$$
\begin{aligned}
p(x+1)-p(x) & =n-k^{2}+2 k+t-2+(2 k-2) x \\
& \geq(k-t+1)^{2}+t-k^{2}+2 k+t-2+(2 k-2)(t+1)>0
\end{aligned}
$$

for each $x \in\{t+1, t+2, \ldots, k-1\}$. Hence $p(x)>0$ for any $x \in\{t+1, t+2, \ldots, k\}$. Let

$$
q(y)=k^{y-t-2}\left(\begin{array}{l}
y \\
t
\end{array}\right)\left(\begin{array}{l}
n-y \\
k-y
\end{array}\right)
$$

for each $y \in\{t+2, t+3, \ldots, k\}$. From $\left(\begin{array}{c}t+2 \\ 2\end{array}\right)(k-t+1)^{2}+t \leq n$ and $p(x)>0$ for any $x \in\{t+1, t+2, \ldots, k\}$, we have

$$
\frac{q(y)}{q(y+1)}=\frac{(y-t+1)(n-y)}{k(y+1)(k-y)}>1
$$

for any $y \in\{t+2, t+2, \ldots, k-1\}$. That is, the function $q(y)$ is decreasing as $y \in\{t+2, t+$ $3, \ldots, k\}$ increases. This together with (14) yields (15) holds.

\section{The proof of Theorem 1.1}

Let $\mathcal{F}$ be any maximal non-trivial $t$-intersecting family which is not given in Theorem 1.1. Suppose $f_{3}(n, k, t)=(f(n, k, t)-|\mathcal{F}|) /\left(\begin{array}{c}n-t-2 \\ k-t-2\end{array}\right)$. It suffices to prove that $f(n, k, t)>|\mathcal{F}|$ or $f_{3}(n, k, t)>0$.

Case 1. $\tau_{t}(\mathcal{F})=t+1$.

Let $\mathcal{T}$ be the set of all $(t+1)$-subsets $T$ of $[n]$ which satisfies $|T \cap F| \geq t$ for any $F \in \mathcal{F}$. Suppose that $|\mathcal{T}|=1$. Since $n \geq\left(\begin{array}{c}t+2 \\ 2\end{array}\right)(k-t+1)^{2}+t$, by Lemma 3.3 (i), we have

$$
f_{3}(n, k, t) \geq n-t-1-\left(\begin{array}{c}
k-t \\
2
\end{array}\right)-(t+1)(k-t)(k-t+1)>0 .
$$


Suppose that $|\mathcal{T}| \geq 2$ and $\tau_{t}(\mathcal{T})=t$. Assume that $l=t+2$. By Lemma 3.3 (ii), we have

$$
f_{3}(n, k, t) \geq \frac{(k-t-2)(n-t-1)}{k-t-1}-\left(\begin{array}{c}
k-t \\
2
\end{array}\right)-(k-1)(k-t+1) .
$$

Observe that $n \geq\left(\begin{array}{c}t+2 \\ 2\end{array}\right)(k-t+1)^{2}+t$. If $k=t+3$, then $f_{3}(n, k, t) \geq 4\left(k^{2}-4 k+2\right)+0.5>0$. If $k=t+4$, then $f_{3}(n, k, t) \geq \frac{5}{3}\left(5 k^{2}-28 k+29\right)>0$. If $k>t+4$, then $n-t-1>$ $(t+2)(k-t+1)(k-t-1)$ and

$$
\begin{aligned}
f_{3}(n, k, t) & >(k-t-2)(t+2)(k-t+1)-(k-1)(k-t+1)-(k-t)(k-t-1) / 2 \\
& =(k-t-4)(k-t+1) t+(k-3)(k-t+1)-(k-t)(k-t-1) / 2 \\
& =(k-t-4)(k-t+1) t+(k-t)(k+t-5) / 2+(k-3)>0 .
\end{aligned}
$$

Assume that $t+2<l<k$. Since $\max \left\{\left(\begin{array}{c}t+2 \\ 2\end{array}\right), \frac{k-t+2}{2}\right\} \geq \frac{1}{k-t}\left(\begin{array}{c}t+2 \\ 2\end{array}\right)+\frac{(k-t-1)(k-t+2)}{2(k-t)}$ and $\left(\begin{array}{c}n-l \\ k-l+1\end{array}\right)<\left(\begin{array}{c}n-t-2 \\ k-t-2\end{array}\right)$, By Lemma 3.3 (ii), then

$$
\begin{aligned}
f_{3}(n, k, t) & >\frac{(k-l)(n-t-1)}{k-t-1}-\left(\begin{array}{c}
k-t \\
2
\end{array}\right)-(k-l+1)(k-t+1)-t \\
& \geq \frac{n-t-1}{k-t-1}-\left(\begin{array}{c}
k-t \\
2
\end{array}\right)-2(k-t+1)-t \\
& \geq \frac{(k-t+1)^{2}}{(k-t)(k-t-1)}\left(\begin{array}{c}
t+2 \\
2
\end{array}\right)-t-1+\frac{(k-t+2)(k-t+1)^{2}}{2(k-t)}-\left(\begin{array}{c}
k-t \\
2
\end{array}\right)-2(k-t+1) \\
& >1+\frac{(k-t+2)(k-t+1)}{2}-\left(\begin{array}{c}
k-t \\
2
\end{array}\right)-2(k-t+1) \geq 0 .
\end{aligned}
$$

Suppose $|\mathcal{T}| \geq 2$ and $\tau_{t}(\mathcal{T})=t+1$. By Lemmas 2.4 and 2.7, we have $f(n, k, t)>|\mathcal{F}|$ if $1 \leq t \leq \frac{k}{2}-\frac{3}{2}$.

Case 2. $t+2 \leq \tau_{t}(\mathcal{F}) \leq k$.

Since

$$
\max \left\{\left(\begin{array}{c}
t+2 \\
2
\end{array}\right), \frac{k-t+2}{2}\right\} \geq \frac{k-t-1}{k-t}\left(\begin{array}{c}
t+2 \\
2
\end{array}\right)+\frac{1}{k-t} \cdot \frac{k-t+2}{2}
$$

by Lemma 3.4, we have

$$
\begin{aligned}
f_{3}(n, k, t) & =\frac{(k-t)(n-t-1)}{k-t-1}-\left(\begin{array}{c}
k-t \\
2
\end{array}\right)-(k-t+1)^{2}\left(\begin{array}{c}
t+2 \\
2
\end{array}\right) \\
& \geq \frac{(k-t+1)^{2}(k-t+2)-2(k-t)}{2(k-t-1)}-\left(\begin{array}{c}
k-t \\
2
\end{array}\right)>0 .
\end{aligned}
$$

Hence the desired result follows.

\section{Acknowledgement}

This research is supported by NSFC (11671043) and NSF of Hebei Province(A2019205092). 


\section{References}

[1] R. Ahlswede and L.H. Khachatrian, The complete nontrivial-intersection theorem for systems of finite sets, J. Combin. Theory Ser. A 76 (1996) 121-138.

[2] R. Ahlswede and L.H. Khachatrian, The complete intersection theorem for systems of finite sets, European J. Combin. 18 (1997) 125-136.

[3] M. Deza and P. Frankl, The Erdős-Ko-Rado theorem-22 years later, SIAM J. Algebraic Discrete Methods 4 (1983) 419-431.

[4] P. Erdős, C. Ko and R. Rado, Intersection theorems for systems of finite sets, Quart. J. Math. Oxf. Ser. (2) 12(48) (1961) 313-320.

[5] P. Frankl, The Erdös-Ko-Rado theorem is true for $n=c k t$, in: Combinatorics, Vol. I, Proc. Fifth Hungarian Colloq., Keszthey, 1976, in: Colloq. Math. Soc. János Bolyai, vol. 18, North-Holland, 1978, pp. 365-375.

[6] P. Frankl, On intersecting families of finite sets, J. Combin. Theory Ser. A 24 (1978) $146-161$.

[7] P. Frankl, On intersecting families of finite sets, Bull. Aust. Math. Soc. 21 (1980) 363372.

[8] P. Frankl, The shifting technique in extremal set theory, in: C. Whitehead (Ed.), Combinatorial Surveys, Cambridge Univ. Press, London, New York, 1987, pp. 81-110.

[9] P. Frankl, Z. Füredi, Nontrivial intersecting families, J. Combin. Theory Ser. A 41 (1986) 150-153.

[10] P. Frankl and Z. Füredi, Beyond the Erdős-Ko-Rado theorem, J. Combin. Theory Ser. A 56 (1991) 182-194.

[11] P. Frankl, K. Ota, N. Tokushige, Covers in uniform intersecting families and a counterexample to a conjecture of lovász, J. Combin. Theory Ser. A 74 (1996) 33-42.

[12] Z. Füredi, Matchings and covers in hypergraphs, Graphs Combin. 4 (1988) 115-206.

[13] M. Furuya, M. Takatou, Covers in 5-uniform intersecting families with covering number three, Australas. J. Combin. 55 (2013) 249-262.

[14] C. Godsil and M. Karen, Erdős-Ko-Rado Theorems: Algebraic Approaches, Cambridge University Press, 2015.

[15] J. Han and Y. Kohayakawa, The maximum size of a non-trivial intersecting uniform family that is not a subfamily of the Hilton-Milner family, Proc. Amer. Math. Soc. 145(1) (2017) 73-87.

[16] A. Hilton and E. Milner, Some intersection theorems for systems of finite sets, Quart. J. Math. Oxford Ser. (2) 18 (1967) 369-384.

[17] A. Kostochka and D. Mubayi, The structure of large intersecting families, Proc. Amer. Math. Soc. 145 (6) (2017) 2311-2321.

[18] R.M. Wilson, The exact bound in the Erdős-Ko-Rado theorem, Combinatorica 4 (1984) $247-257$. 